\title{
THE INFLUENCE OF HEART STRESS ON THE BRAIN: A PRELIMINARY STUDY
}

\author{
A.K. MACPHERSON ${ }^{1}$, S. NETI ${ }^{1}$, M. AVERBACH ${ }^{2}$, P.A. MACPHERSON ${ }^{3}$, C. CHUTAKOSITKANON ${ }^{1}$ \\ \& D. NATHANSON ${ }^{2}$ \\ ${ }^{1}$ Institute of Biomedical Engineering and Mathematical Biology, Lehigh University, USA. \\ ${ }^{2}$ Division of Cardiology, St Lukes Hospital, USA. \\ ${ }^{3}$ Department of Applied Technology, Rogers State University, USA.
}

\begin{abstract}
The interaction between the cardiac hemodynamics and brain activity has been extensively studied in the past. However these studies have involved not only an increase in heart rate but also an increase in blood pressure. One method to evaluate for underlying coronary artery disease is to perform stress testing. Often, myocardial stress is achieved by the patient walking on a treadmill or riding a stationary bicycle while being monitored. In patients that are unable to exercise, pharmacologic stress testing is performed, either with vasodilatory agents (e.g. adenosine) or dobutamine, which is a pro-inotropic and chronotropic drug. During dobutamine infusion, the heart rate increases, but there is a negligible increase in blood pressure. The result is that the heart rate increases as a function of the dosage. Thus by monitoring brain activity while patients are undergoing dobutamine stress testing the effect of increased blood pressure on the brain is removed. It was found in the results of one patient that there are five areas of the brain which show a change in the frequency of EEG activity that is a function of dobutamine dosage. Using Fourier transforms it was established that this change occurred at a frequency around $12.5 \mathrm{~Hz}$. The regions of the brain where the increased activity were a function of heart rate occurred in the CZ-PZ, C4-P4, T5-O1, T4-T6 and C3-P3 regions. In undertaking these calculations it was realized that the stress on the ventricle could be calculated. The physicians monitor ECG of the patient to evaluate the cardiac status of the patient. Since currently the attending physician does not have data on the stress being applied to the myocardium, it would be useful to have this calculated on a real time basis. The motivation for the present preliminary study is to both investigate the feasibility of producing such information for clinicians as well as to investigate the variation between different patients as the heart rate varies. It was found that generally the surface shear stress increased with heart rate around most of the left ventricle. While the time averaged shear stress may be important for diagnosis the maximum shear stress probably is the limiting factor in terminating testing.
\end{abstract}

Keywords: brain, diagnostic tool, echocardiogram, electroencephalogram, frequency, heart, stress.

\section{INTRODUCTION}

The physically verified data on the interaction of the heart and brain is rather limited. It has not been possible to identify specific parts of the brain which results in specific actions of the heart. It may be that this is not the way the heart and brain interact. Such a conclusion is reached in [1]. An extensive review of the key experiments on how the brain regulates the cardiovascular system are examined. From this review it does not appear that there is sufficient compelling evidence to determine exactly which portions of the brain controls the heart. The control of the muscle fibers in the wall of blood vessel provides an indication of the difficulty of reaching final conclusions on the brain-heart interaction. It was discovered in the 19th century that there existed a vasomotor system of nerve fibers which controlled the muscle fibers in the wall of blood vessels. Today it is still unclear whether the spontaneous activity in the vasomotor nerves arises from the intrinsic activity of pacemaker cells or from assemblies of neurons. In addition it is suggested that the hypothalamus plays a significant role in different vascular responses. There are other open questions such as in heart failure [1] 'Is it a signal from the heart to the brain that results in the CNS changes or are there changes occurring first in the supramedullary regions of the brain?' Based on the ability of the body to deliver blood to 
organs where the blood is needed the idea of command neurons is suggested. This could lead to the brain being the cause of hypertension and congestive heart failure.

There are some areas where the interaction is fairly well established. One is a system termed the baroflex which controls the arterial blood pressure. It is suggested in [2] that a complete physical description of the various components of the baroflex have been developed. As an understanding, based on physical observation, of the behavior of the baroflex is unknown a mathematical model is proposed in [2]. Based on that model it was shown there is considerable feedback between the pressure regulators and the heart rate. The interaction occurs both through the parasympathetic and the sympathetic systems.

An experiment close to the present work was undertaken in [3]. The objective of the study was to construct a model for determining the effect of stress on patients. As part of the study, physical load was induced in a subject by exercising on a bicycle. An extensive monitoring system of blood pressure, heart rate, respiration, body temperature, and electroencephalography (EEG) signals was setup. Although only one EEG recording site is mentioned in the paper it appears that a number of sites were recorded. The conclusions reached were ' Two circumscribed clusters of significant association between heartbeat-evoked potential (HEP) and cardiac output change (independent of changes in systolic blood pressure) were observed in left temporal and lateral frontal regions. We observed no overall alteration across electrodes in the amplitude of HEP (455-595 ms after R wave) as a function of mental stress'. This result suggests that a specific connection between heart rate and two regions of the brain were established. Unfortunately due to the exercise there were increases in blood pressure, temperature and respiration rate as well as heart rate. The analysis of the test results of 16 subjects indicated that these factors did not influence the heart-brain interaction. However for the purpose of the study, the tests were undertaken on healthy subjects. In a hospital environment involving a wide variety of patients, the results may be different. In [4] it was found on testing 10 patients with average age 59 years and all suffering from some heart disease that 'Across patients, HEP amplitude at left temporal and lateral frontal electrode locations correlated with stress-induced changes in cardiac output, consistent with an afferent cortical representation of myocardial function during stress'. It is known that the effects of shear stress on the heart are very extensive. Two surveys $[5,6]$ showed that stress can cause changes in the genetic structure of the heart.

Dobutamine acts to increase the heart rate and dilates the arteries. Thus the blood pressure does not increase so the baroreceptors do not transmit signals to the brain. In addition, the temperature does not change as in the standard exercise stress test. This provides a clean experiment where only the motion of the ventricle transmits signals to the brain. Thus the possible effects of these variables are removed from the results. This is achieved by the injection of dobutamine. Under conditions where, due to reduced physical capability, patients cannot undertake the usual exercise stress test, then the heart is stressed by dobutamine injections at various rates. For the purposes of analysis the results obtained are superior to those obtained from an exercise stress test as dobutamine dilates the arteries neither the patient's blood pressure nor temperature increases.

\section{METHODS OF EXPERIMENTS AND CALCULATION}

A patient who was scheduled to undergo a dobutamine stress test was instrumented with 19 electrodes in the standard positions on the scalp (Fig. 1). At the same time, ECG results were obtained while the dobutamine was administered. There were results from the use of three different dosages of dobutamine. These were 0,1560 and $2750 \mathrm{mics} / \mathrm{min}$. As the usual designation of the injection is 20 and $35 \mathrm{mics} / \mathrm{kg} / \mathrm{min}$ these designations have been used below. In order to establish any frequency changes due to dobutamine the EEG results were analyzed using MATLAB to obtain the Fourier transforms. 

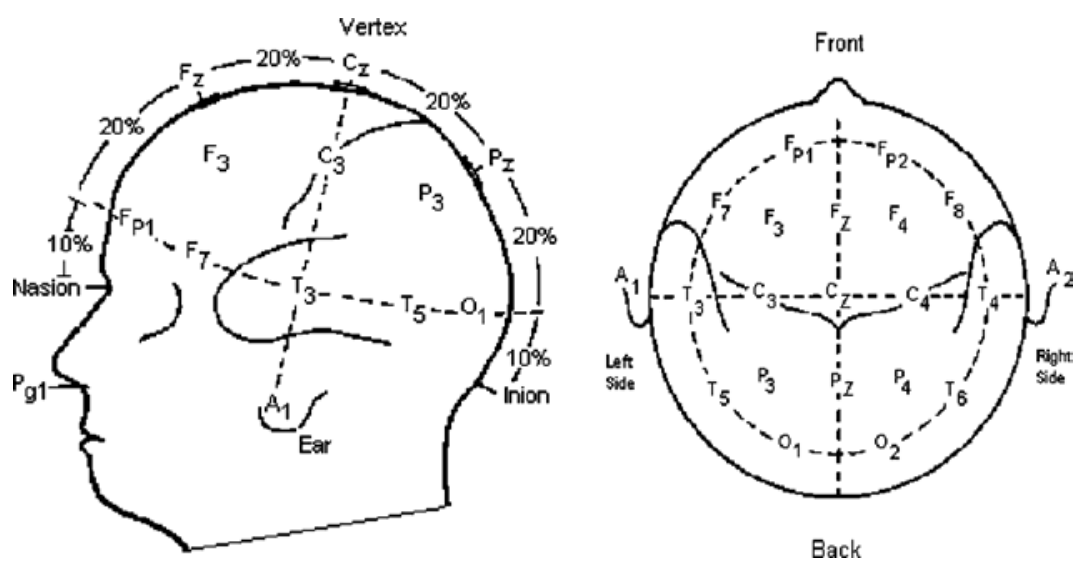

Figure 1: Electrode positions on skull. From website by Eric Chudler, University of Washington.

It was of interest to establish the stress level on the heart at each dosage. This was undertaken by firstly calculating the blood flow. The general method of calculation used here has been described previously [7-9]. The solution is in two dimensions. In the solution the blood flow into the left atrium is simulated by a source distributed throughout the atrium. In order to conserve mass sinks are distribute around the periphery of the integration domain. The change in shape is obtained from the echocardiograms and used as boundary conditions for the flow. The source strength has to match the change in volume of the ventricle. The valves have to be modeled as thicker than in reality as Lagrangian integration must go around both sides of the valve. The Navier-Stokes equations are then solved with a predictor corrector scheme [7].

The Navier-Stokes equations defined on an $x-y$ Cartesian co-ordinate system for an incompressible fluid are

$$
\rho\left(\frac{\partial \hat{u}}{\partial t}+\hat{u} \cdot \nabla \hat{u}\right)+\nabla p=\mu \nabla^{2} \hat{u}+\hat{F}
$$

$$
\nabla \hat{u}=0
$$

where $\hat{u}$ is the blood velocity vector, $\rho$ the density, $t$ the time, $p$ the pressure, and the viscosity is $\mu$.

The boundary force $\hat{F}$ arising from the heart muscles is

$$
\hat{F}(\hat{x}, t)=\int_{0}^{L} \hat{f}(s, t) \delta(\hat{x}-\hat{X}(s, t)) d s
$$

Here $\hat{f}$ is the force on the boundary element at the point $s$ defined on a Lagrangian system where $\hat{x}$ is defined on the Cartesian system and $\hat{X}^{n}$ is the $n$th point on the Lagrangian system, $L$ the circumference of the ventricle. If $\hat{f}$ is known then it would be used. In the present case the value of $\hat{f}$ varies with time and position on the ventricle. The value of $\hat{f}$ can be obtained by using the difference equation [7].

$$
x_{k}^{*}=\left[x_{k}^{n}+(\Delta t) u_{k}^{n}\right]+\lambda \hat{f}_{\mathrm{k}}
$$


where $k$ is a Cartesian grid point, the position $x_{k}{ }^{*}$ is the value of $x_{k}^{n}$ at the next time step, $u$ is the velocity and $\lambda$ is a complex function of the source strength and a function to produce a linear resistance model of the blood flow through the arteries. $\lambda$ is also dependent on the strength of the sinks external to the ventricle.

$$
\lambda=\frac{9\left(\Delta t^{2}\right) \beta}{64 h^{2} N}
$$

where $\beta$ is an adjustable parameter of order $1, h$ is the Cartesian grid spacing and $N$ is the number of boundary points. The delta function is defined as

$$
\begin{gathered}
\delta_{k}=\delta(x) \delta(y) \\
\delta(x)=\frac{1}{4 h}\left(1+\cos \frac{\pi x}{2 h}\right) \quad \text { if }|x| \leq 2 h \text { otherwise } \delta^{k}=0
\end{gathered}
$$

From echocardiograms of the patient the positions of the wall can be measured at various times. Thus the velocity of the wall can be calculated and hence $\hat{f}_{k}$ can be obtained. This value of $\hat{f}$ is both time dependent and spatially dependent.

The flow velocities and pressures can be used to calculate the stresses on the surface of the heart walls. These forces can then be used to examine the microscopic interaction with the cells in the heart wall (endocardium).

The first step in the solution involves obtaining the shape of the ventricle at various times. This is often difficult as echocardiogram images are sometimes indistinct. Following a method often used by echocardiographers only five images in a cardiac cycle were selected. One image when the valves were closed, a second image when the valves were fully open, a third just before the atrium starts to contract, one at the end of the ventricle filling (diastole) stage and a final one as the aortic valve opens. A linear variation was assumed between each image, time frame. It was assumed that the motion of the wall would be normal to the surface.

The second step required the simulation of the atrium. The atrium changes shape during the diastole stage and thus changes the pressure. However the use of a source in place of the correct inflow pattern to the atrium was an artifice which made the actual atrium shape unimportant. The atrium shape was fixed at near hemispherical shape with valves in the closed and early open positions. After some time the atrium contracts for a period before the mitral valve closed. The shape was expanded and contracted as required for the different sized mitral valves. The source strength was increased slowly as the valves opened in accordance with the increase in volume of the ventricle.

The stresses at the walls were calculated by selecting two points chosen as close to the wall as possible along a line normal to the surface. A finite difference method was used to obtain the derivative of the velocity along this line. Similarly the velocity normal to the wall was calculated along the same line. Thus if $y$ is normal to the wall the fluid shear stress $\tau_{0}$ is calculated to provide $\tau=\mu \partial u / \partial y$ and the coupling to the wall is obtained from $\mu d^{2} u / d^{2} y=K u$ where $K$ is the hydraulic resistivity of the wall.

\section{RESULTS}

The electrode traces after the first injection of dobutamine are shown in Fig. 2.

It can be seen that there were eye movement amplitude changes detected by the frontal electrodes.

The electrode traces after 35 mics of dobutamine are shown in Fig. 3. 


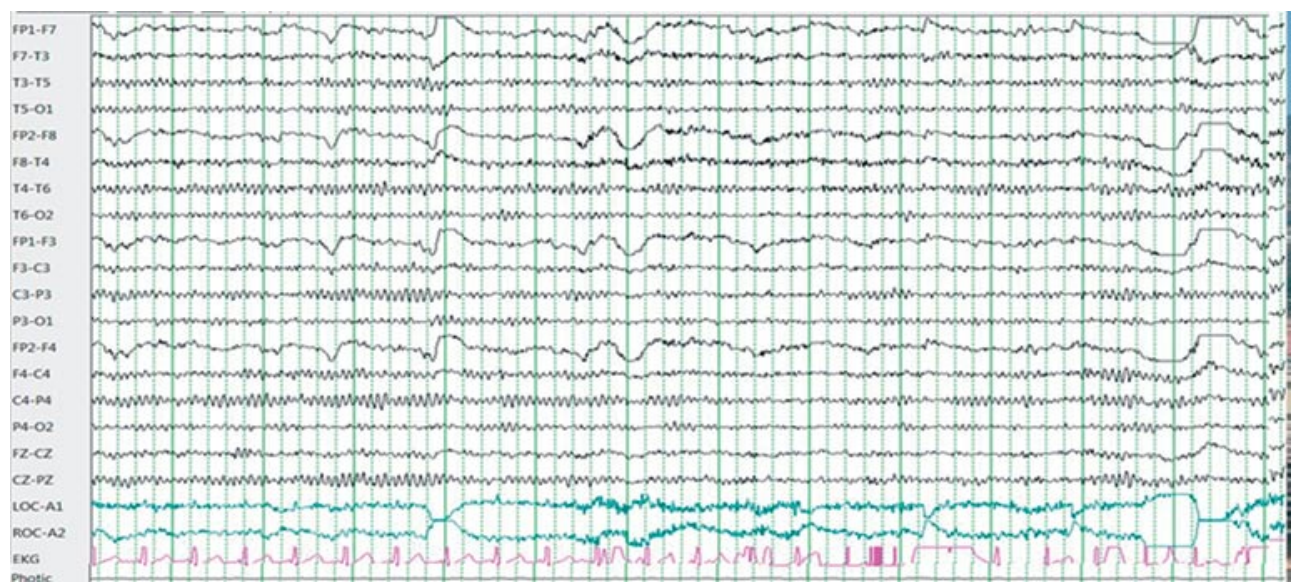

Figure 2: Electrode traces after injection of 20 mics of dobtamine.

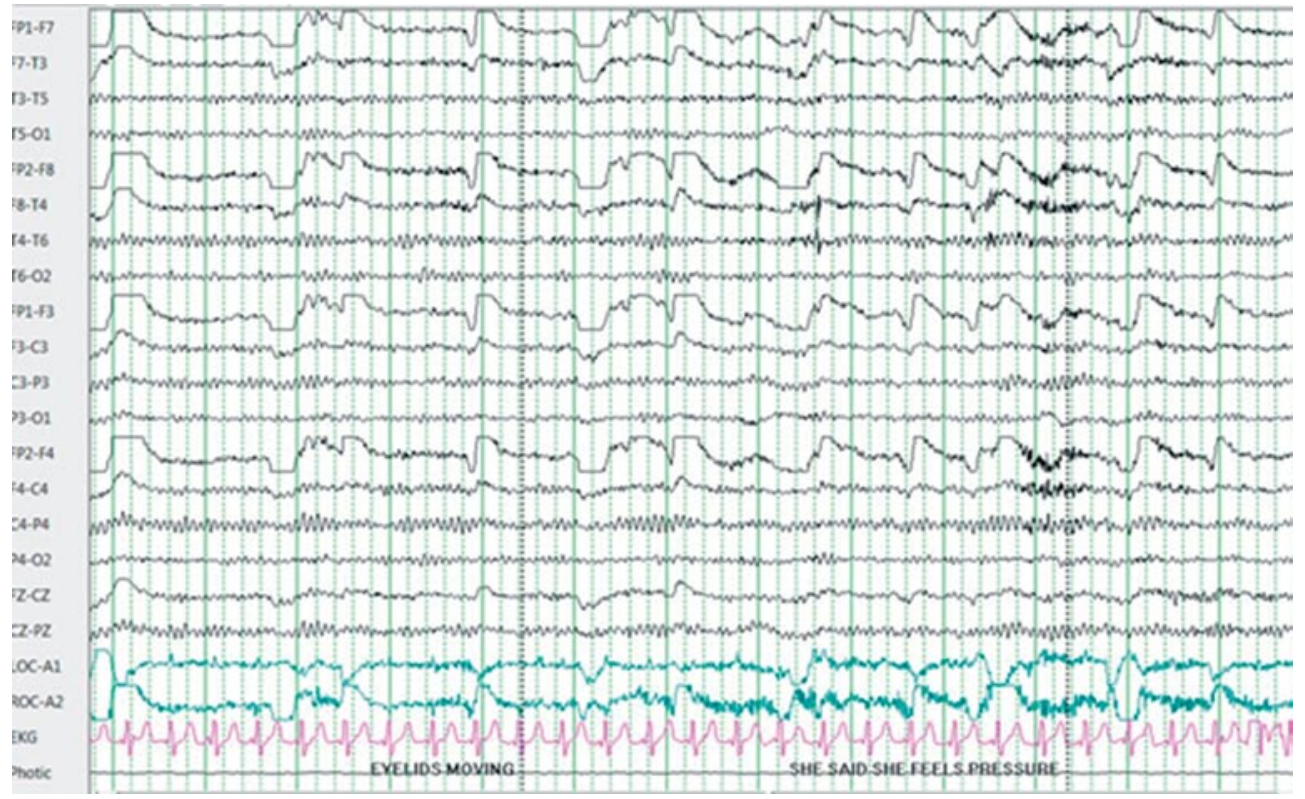

Figure 3: Electrode traces after injection of 35 mics of dobtamine.

By observation it was thought that there was a change in frequency in some of the transducers output. In order to examine this, Fourier transforms were performed on all of these results except for the ear tracings LOC-A1 and ROC-A2. The relevant results are shown in Figs 4-7 where the absolute values of the signals are shown.

Figure 4 is on the right just above the eye level swinging back from the ears.

Figure 5 is on the right just above the eye level. It is in a similar position to Fig. 4 but more to the center of the head. Again it moves to the rear of the skull. 

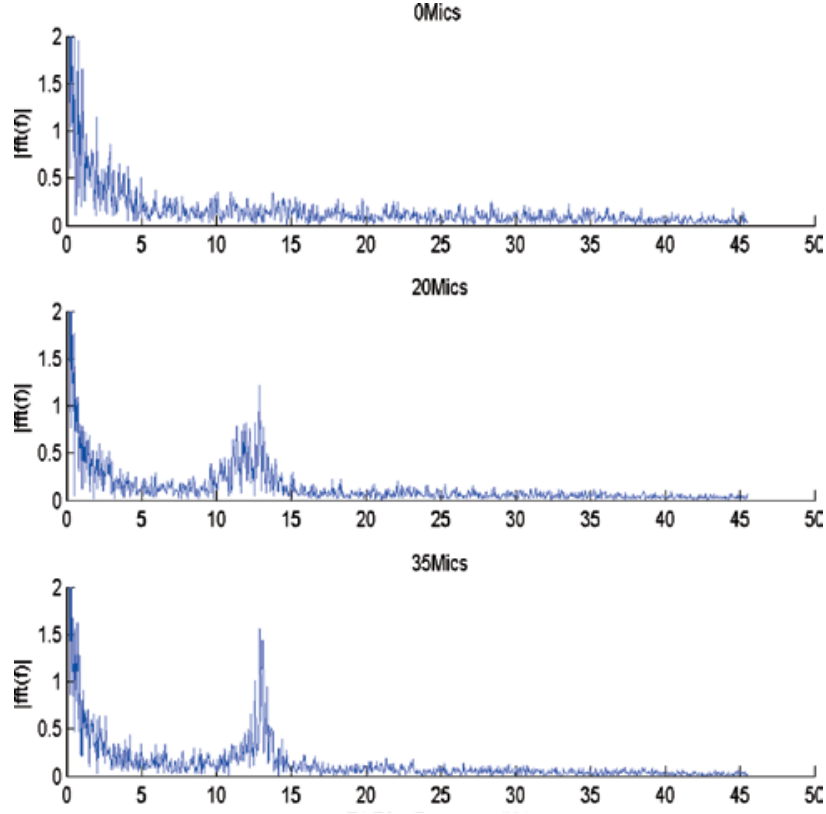

Figure 4: Fourier transforms of electrode traces at the T4-T6 (Fig. 1) location after varying injections of dobutamine.
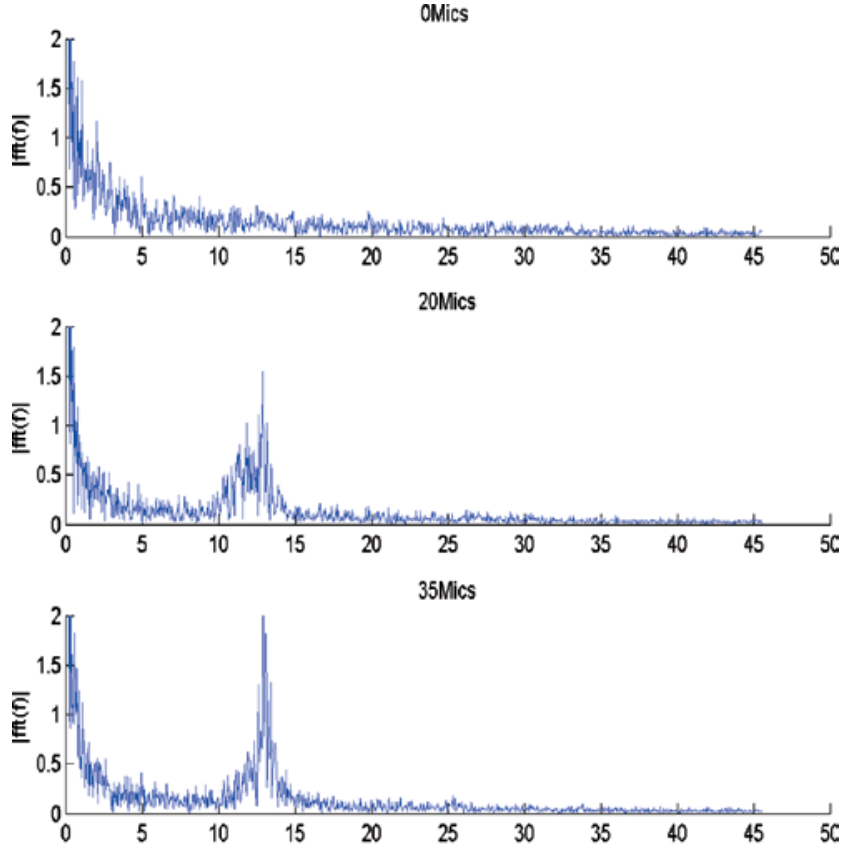

Figure 5: Fourier transforms of electrode traces at the C4-P4 (Fig. 1) location after varying injections of dobutamine. 

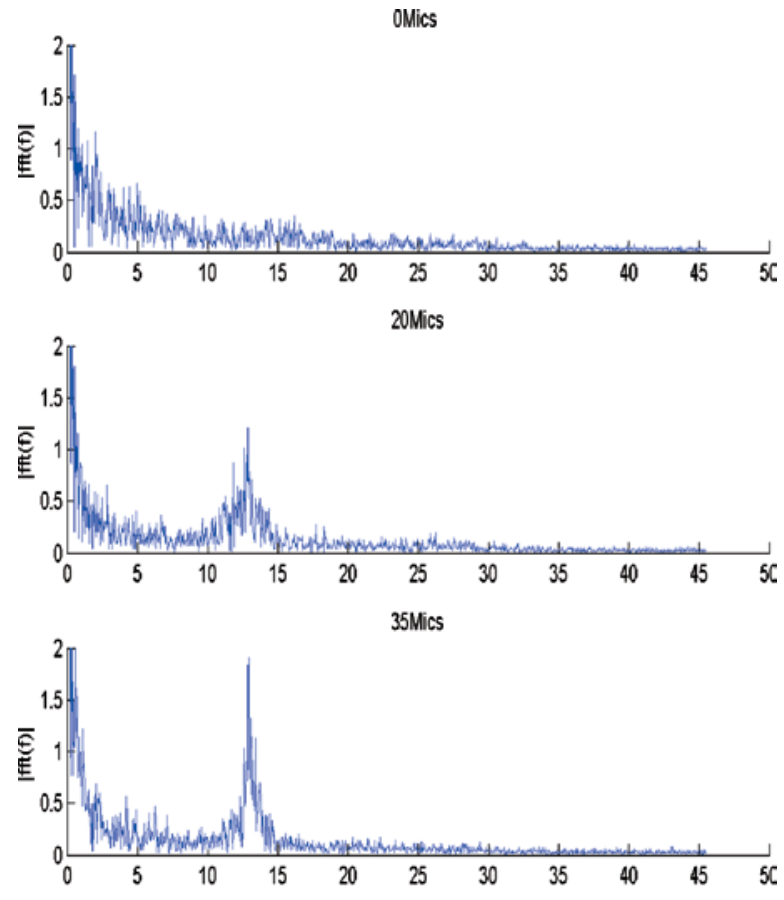

Figure 6: Fourier transforms of electrode traces at the CZ-PZ locations (Fig. 1) after injection of varying injections of dobutamine.
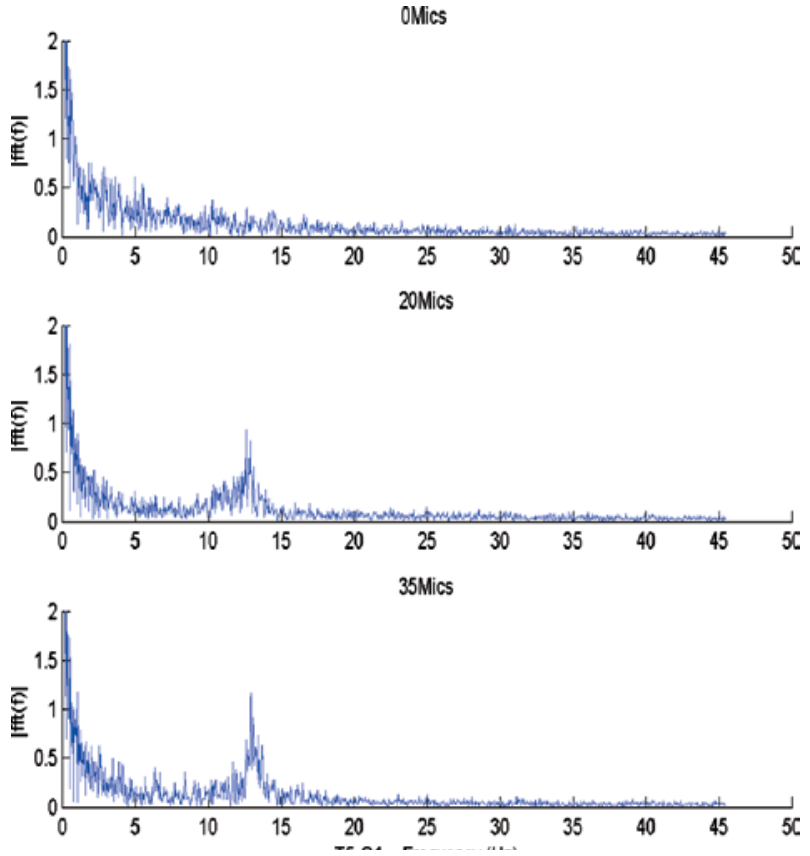

Figure 7: Fourier transforms of electrode traces at locations T5-O1 locations (Fig. 1) after injection of varying injections of dobutamine. 

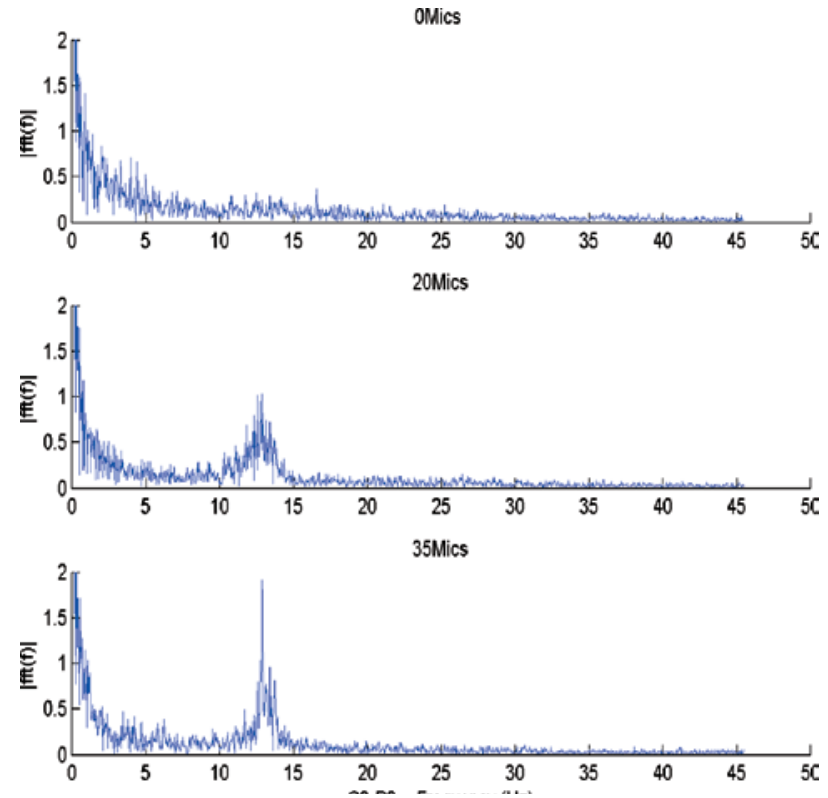

Figure 8: Fourier transforms of electrode traces at the C3-P3 (Fig. 1) location after varying injections of dobutamine.

Figure 6 is running from the top of the skull backwards to a similar extent as the T6 location.

Figure 7 is on the left side of the skull and extends backwards. In this case it starts on the opposite side of the skull at a location similar to $\mathrm{T} 6$.

Figure 8 is on the left just above the eye level but further towards the center of the head. It a mirror position of Fig. 5.

It can be seen from Figs 4-8 that the power of the transform has a peak at a frequency close to $12.5 \mathrm{~Hz}$ that is the normal alpha rhythm. The locations in Figs 5 and 8 are towards the center of the skull. The signals from the right side appear stronger than the left. The center location Fig. 6 also shows a strong signal. The outer locations in Figs 5 and 7 do not show such strong transforms. Thus it would appear that the significant signal strength is found on the right side of the head arising towards the center of the skull. Certainly additional results are required to reach definite conclusions of its significance. It is interesting, although from only one result not necessarily significant, that the Fourier transform power has a peak at almost all locations at the same frequency.

Although the dosage of dobutamine is relevant to the load on the heart, the important variable in assessing the neural signals is probably the stress on the heart. In calculating this data it was realized that it could be expressed in a number of ways. One way is to show the variation of both the maximum and average shear stress over the ventricle as a function of time as shown in Fig. 9. The average was calculated over the regions shown in thick lines. For example the endocardium average shear stress is obtained by averaging the Lagrangian locations 5-9.

The results were obtained for three patients. Patient 1 was a 55 year old woman, Patient 2 was a 75 year old woman and Patient 3 was a 75 year old woman. The results in Figs 10 and 11 were for Patient 1. 


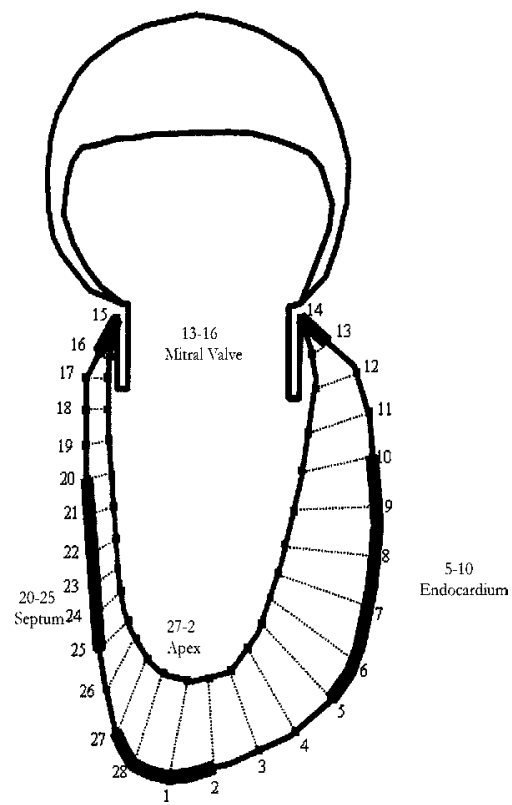

Figure 9: Areas over which averages taken.

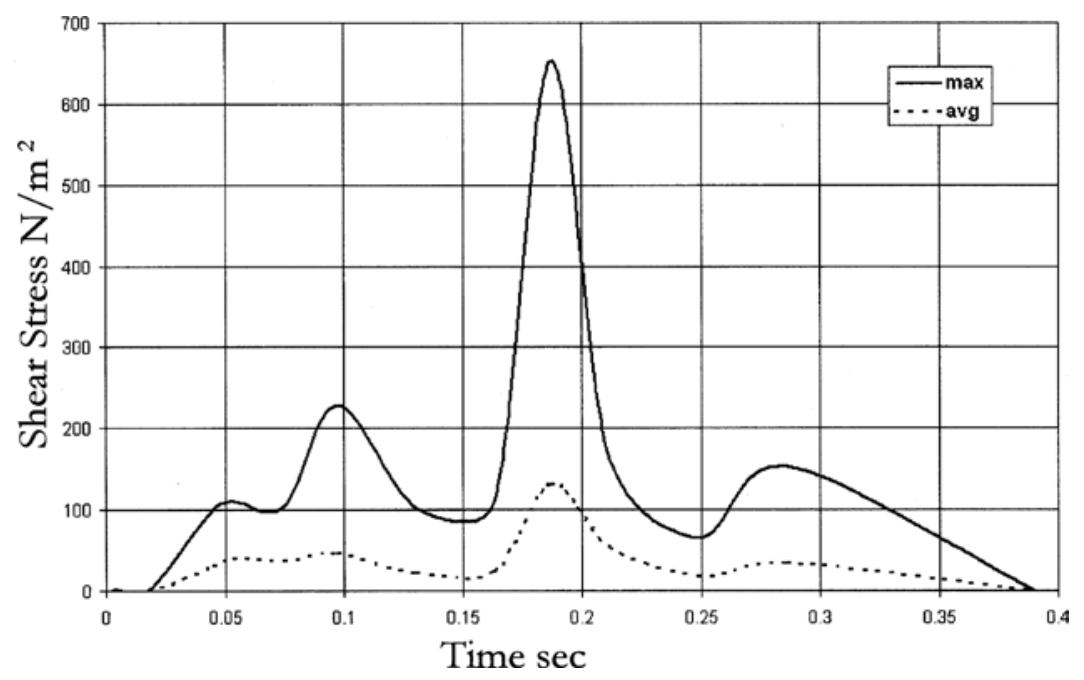

Figure 10: Variation of maximum and average shear stress over the ventricle.

It can be seen that the maximum occurs approximately when the mitral valves are open.

The maximum shear stress in the apex occurs just as the mitral valve is opening.

The shear stress over the apex is larger than that over the endocardium. However it is known that failure most often occurs in the endocardium. Further, sometimes the stress over the endocardium decreases with heart rate as shown in Fig. 12(c) whereas a patient feels stress as the heart rate increases. Thus the maximum shear stress at any point on the ventricle was examined as in Fig. 13. 


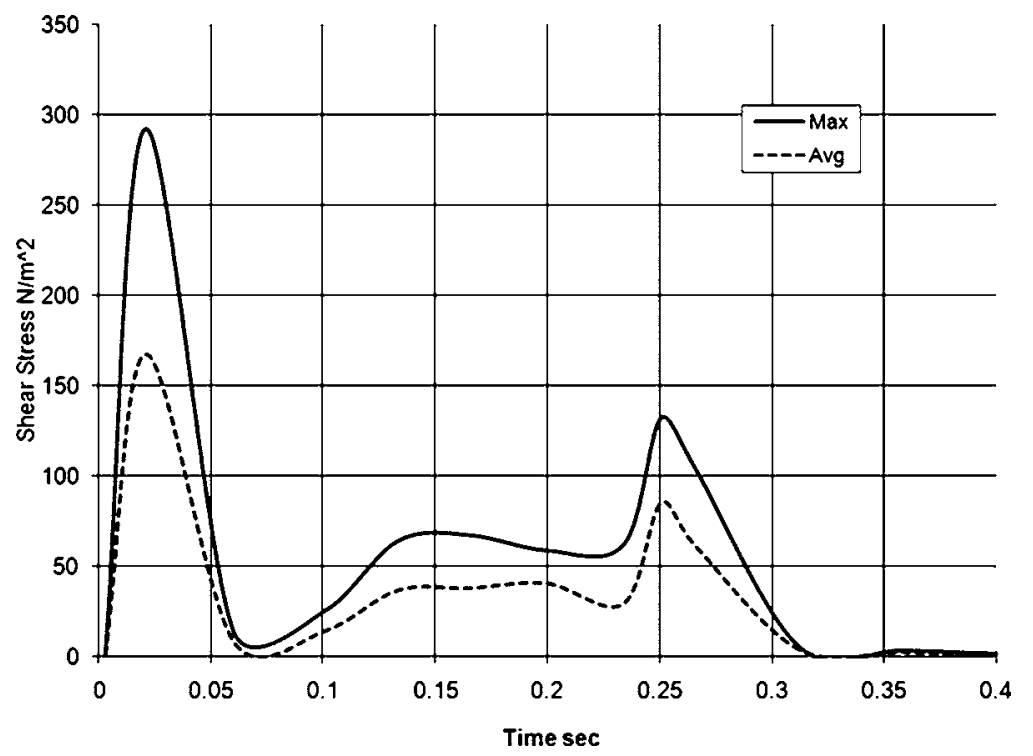

Figure 11: Variation of maximum and average shear stress over the apex.

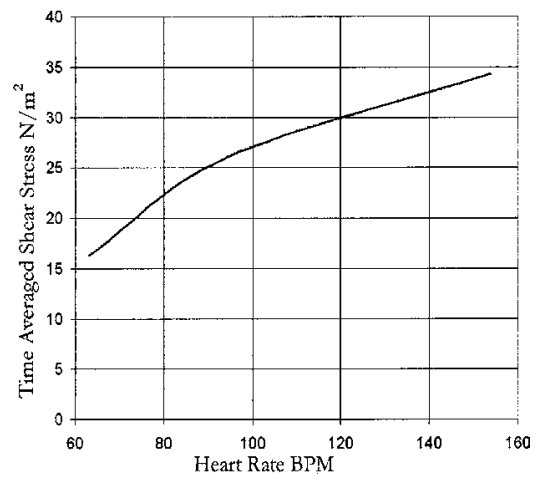

(a)

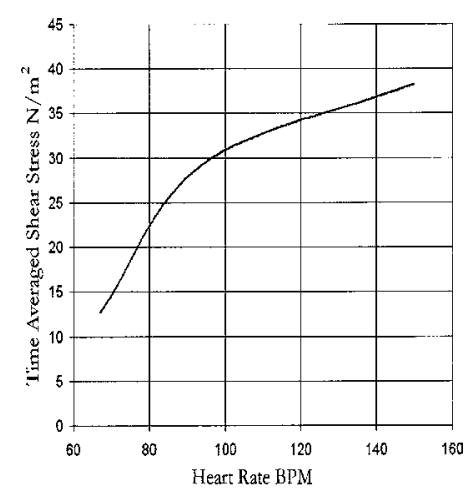

(b)

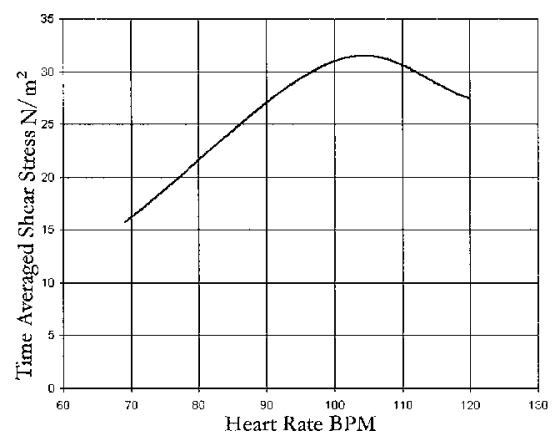

(c)

Figure 12: Variation of time averaged endocardium shear stress over on heart beat as a function of heart rate. (a) Patient 1, (b) Patient 2, (c) Patient 3. 


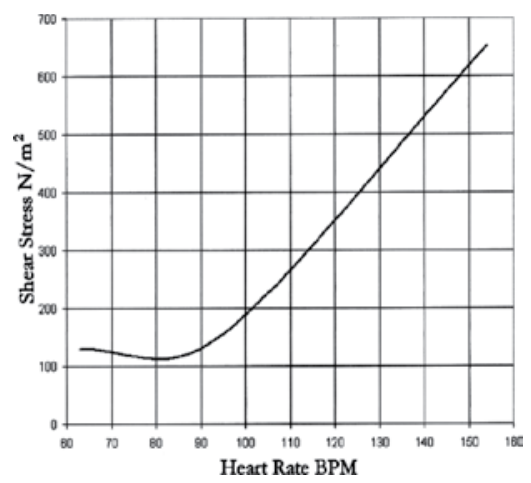

(a)

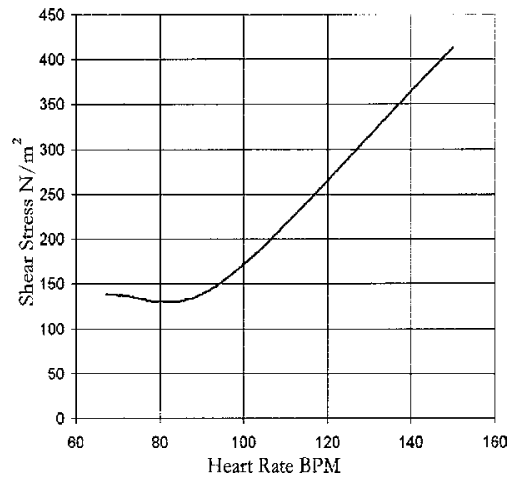

(b)

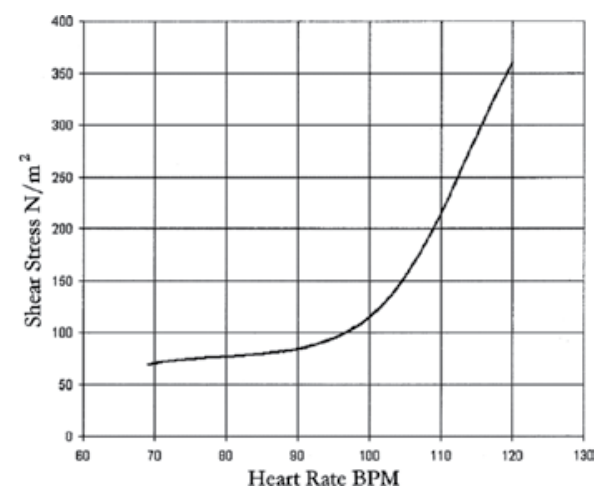

(c)

Figure 13: The Variation of the maximum shear stress on left ventricle with heart rate. (a) Patient 1, (b) Patient 2, (c) Patient 3.

It can be seen that the maximum shear stress shown in Fig. 12 increases rapidly as a function of heart rate and would appear to be the significantly important variable. It was realized that this data if calculated on a real time basis would be a useful input to a physician in deciding when to terminate the test. The measurement of surface shear stress would be very difficult and values are not available. Thus it is not possible to know the critical shear stress for a given patient. However for the proposed purpose the calculated values would be adequate.

\section{CONCLUSIONS AND FUTURE WORK}

It has been shown that there is a definite relation between heart stress and certain brain activity. As there was only one set of data available general conclusions cannot be drawn. It is intended to continue the study with additional patients. This preliminary study has shown that data useful to a clinician monitoring dobutamine testing can be obtained. In addition, diagnostic results can be extracted from the data. It is intended to continue the study with additional patients in order to draw general conclusions.

\section{ACKNOWLEDGEMENTS}

We would like to acknowledge the assistance of a Lehigh student Kristy Caltabiano in setting up the initial MATLAB calculations. 


\section{REFERENCES}

[1] John, H.C., Landmarks in understanding the central nervous control of the cardiovascular system. Experimental Physiology, 92.1 pp. 3-18.

[2] Mauro, U., Interaction between carotid baroregulation and the pulsating heart: a mathematical model. American Journal of Physiology. Heart and Circulatory Physiology, 275, pp. 1733-1747, 1998.

[3] Emad, El-S., Mahdi, M., Derek, A.L., A closed-loop hybrid physiological model relating to subjects under physical stress. Artificial Intelligence in Medicine, 38, pp. 257-274, 2006. doi:10.1016/j.artmed.2006.07.009

[4] Marcus, A.G., Peter, T., Peter, M.S., David, G., Diana, R.H., David, B., David, B. \& Hugo, D.C., A cortical potential reflecting cardiac function. PNAS, 104(16), pp. 6818-6823, 2007. doi:10.1073/pnas.0609509104

[5] Sadoshima, J. \& Izumo, S., The cellular and molecular response of cardiac myocytes to mechanical stress. Annual Review of Physiology, 59, pp. 551-571, 1997. doi:10.1146/annurev. physiol.59.1.551

[6] Ruwhof, C. \& van der Laarse, A., Mechanical stress-induced cardiac hypertrophy:mechanisms and signal transduction pathways. Cardiovascular Research, 47, 23-37, 2000. doi:10.1016/ S0008-6363(00)00076-6

[7] Macpherson, A.K. \& Neti, S., The effect of angiotensin II on heart blood flow and hypertension. Advances Fluid Mechanics IV, eds M. Rahman, R.Verhoeven \& C.A. Brebbia, WIT Press: Southampton, pp.1-12, 2002.

[8] Macpherson, A.K., Neti, S., Macpherson, PA., Houser, SR., Hari, M. \& Marzillier, J., Mechanical stress and hypertrophy. Modelling in Medicine and Biology VI, eds M. Ursino, Ca.a.Brebbia, G. Pontrelli, \& E. Magasso, WIT Press: Southampton, pp. 171-179, 2005.

[9] Macpherson, A.K., Neti, S., Chutakostikanon, C., Averbach, M. \& Macpherson, P.A., The variation of dobutamine induced heart stress with heart rate. Modelling in Medicine and Biology VI, ed C.A. Brebbia, WIT Press: Southhampton, pp. 17-26, 2009.

The following comment has been provided by an anonymous reviewer.

Traditionally, echocardiography and other imaging modalities have provided the ability to measure radial motion of the myocardium on a regional or global basis. The development of strain and strain rate techniques has provided the ability not only to assess but also to quantify longitudinal motion (i.e. ventricular shortening).

More recent literature indicated a possible role of adenosine stress MR (magnetic resonance) perfusion (magnetic resonance perfusion - MRP) imaging in identifying patients at high risk for future cardiac events; however, as for dobutamine stress MR - DSMR. Cardiac MR imaging offers the advantage of conducting myocardial perfusion and wall motion measurements at rest and under stress conditions during a single-session examination, thereby ensuring superb comparability of test results [1].

Because many patients are unable to perform adequate exercise testing because of physical incapacity, dobutamine stress echo (DSE) has become a well-established modality for the diagnosis of myocardial ischemia.

Dobutamine stress echocardiography is used widely to assess coronary artery disease (CAD), its interpretation is demanding, and its accuracy depends on the experience and training of the reader [2]. Despite technical advances and digital image processing and display, both its reproducibility and accuracy are dependent on image quality. DSE also is limited by its relative insensitivity for mild single-vessel disease, low sensitivity for recognizing the presence of multivessel coronary disease, and difficulty in detection of ischemia within areas of abnormal resting wall motion (WM).

Quantification of DSE with tissue Doppler is less dependent on 2-dimensional image quality than quantification from 2-dimensional echocardiography, and several studies have shown the technique to be feasible for DSE using 
either velocity or strain rate imaging (SRI) [3]. SR and strain can characterize regional myocardial deformation at rest and can quantify normal or abnormal regional function during DSE [4].

Conventional DSE are two important problems. First, it remains a subjective tool; its accuracy depends on the skills of the user. Second, although the extent and severity of abnormality at peak stress are predictive of outcome [5], WM abnormality is known to be relatively poor for distinguishing the true extent of CAD.

A recent review of 28 major DSE studies [6] has reported sensitivity, specificity, and accuracy ranging from 54 to $96 \%, 60$ to $100 \%$, and 62 to $92 \%$, respectively, depending on patient selection, study size, definition of coronary artery disease and technical equipment.

Connection between the nervous system and the cardiopulmonary system provides the unifying link that allows a coherent explanation for most, if not all, of the forms of neurocardiac damage. It has been known for a long time that stimulation of the hypothalamus can lead to autonomic cardiovascular disturbances [7], and many years ago lesions in the heart and gastrointestinal tract have been produced with hypothalamic stimulation.

Brain natriuretic peptide is produced by the ventricular myocardium in response to stretch; its vasodilator and natriuretic effects counteract the opposing actions of angiotensin II and aldosterone [8].

Increased levels of circulating neurohormones are only part of the response seen after an initial insult to the myocardium. Left ventricular remodeling is the process, by which mechanical, neurohormonal, and possibly genetic factors alter ventricular size, shape, and function.

\section{REFERENCES}

[1] Paetsch, I., Jahnke, C., Wahl, A., Gebker, R., Neuss, M., Fleck, E. \& Nagel, E., Comparison of dobutamine stress magnetic resonance, adenosine stress magnetic resonance, and adenosine stress magnetic resonance perfusion. Circulation, 110, pp. 835-842, 2004. doi:10.1161/01.CIR.0000138927.00357.FB

[2] Picano, E., Lattanzi, F., Orlandini, A., Marini, C. \& L'Abbate, A., Stress echocardiography and the human factor: the importance of being expert. Journal of the American College of Cardiology, 17, pp. 666-669, 1991.

[3] Madler, C.F., Payne, N., Wilkenshoff, U., Cohen, A., Derumeaux, G.A., Pierard, L.A., Engvall, J., Brodin, L.A., Sutherland, G.R. \& Fraser, A.G., Non-invasive diagnosis of coronary artery disease by quantitative stress echocardiography: optimal diagnostic models using off-line tissue Doppler in the MYDISE study. European Heart Journal, 24, pp. 1584-1594, 2003. doi:10.1016/S0195-668$\mathrm{X}(03) 00099-\mathrm{X}$

[4] Voigt, J.U., Exner, B., Schmiedehausen, K., Huchzermeyer, C., Reulbach, U., Nixdorff, U., Platsch, G., Kuwert, T., Daniel, W.G., Flachskampf, F.A., Strain-rate imaging during dobutamine stress echocardiography provides objective evidence of inducible ischemia. Circulation, 107, pp. 2120-2126, 2003. doi:10.1161/01.CIR.0000065249.69988.AA

[5] Hoffman, R., Lethen, H., Kuhl, H., Lepper, W. \& Hanrath, P., Extent and severity of test positivity during dobutamine stress echocardiography. European Heart Journal, 20, pp. 1485-1492, 1999. doi:10.1053/euhj.1999.1628

[6] Geleijnse, M.L., Fioretti, P.M. \& Roelandt, J.R., Methodology, feasibility, safety and diagnostic accuracy of dobutamine stress echocardiography. Journal of the American College of Cardiology, 30, pp. 595-606, 1997.

[7] Dikshit, B.B., The production of cardiac irregularities by excitation of the hypothalamic centres. Journal of Physiology, 81, pp. 382-394, 1934. doi:10.1016/S0735-1097(97)00206-4

[8] Jessup, M., Brozena, S., Heart failure. The New England Journal of Medicine, 348, pp. 2007-2018, 2003. doi:10.1056/NEJMra021498, doi:10.1056/NEJMe030030 